\title{
Modern meningioma imaging techniques
}

\author{
D. Saloner $\cdot$ A. Uzelac $\cdot$ S. Hetts $\cdot$ A. Martin $\cdot$ \\ W. Dillon
}

Received: 2 July 2010/ Accepted: 17 August 2010/Published online: 1 September 2010

(C) The Author(s) 2010. This article is published with open access at Springerlink.com

\begin{abstract}
Steady improvements in imaging modalities have enabled a new realm of capabilities in the identification and assessment of meningiomas. The cross-sectional imaging modalities, MRI and CT, have improved in resolution and fidelity. These modalites now provide not only improved structural information but also insights into functional behavior. MRI has, in particular, proven to have powerful capabilities in evaluating meningiomas because of the ability to assess soft tissue characteristics such as diffusion and vascular supply information, such as perfusion. Recent investigational advances have also been made using a combination of X-ray fluoroscopy for selective catheterization followed by MR perfusion measurement performed with intra-arterial injection of contrast. Together all these modalities provide the radiographer with powerful capbilities for evaluating meningiomas.
\end{abstract}

Keywords Meningioma $\cdot$ MRI $\cdot$ CT $\cdot$ Angiography Perfusion · Imaging

\section{Introduction}

The detection and accurate diagnosis of meningiomas has been dramatically improved by the availability of modern cross-sectional imaging methods, namely magnetic resonance imaging (MRI) and multi-detector computed tomography (MDCT). Not only do these modalities provide highly detailed information on the structure and composition of meningiomas but also important insights into functional

D. Saloner $(\varangle)$ - A. Uzelac $\cdot$ S. Hetts · A. Martin · W. Dillon Department of Radiology and Biomedical Imaging, University of California, San Francisco, CA, USA

e-mail: saloner@radmail.ucsf.edu aspects of the tumor. Once a meningioma has been identified and surgery is planned, imaging plays an essential role when embolization is performed utilizing X-ray fluoroscopy to reduce blood loss at subsequent surgery.

\section{Computed tomography}

Although MRI is the imaging study of choice for evaluation of suspected meningioma or in the context of known or highly suspected pathology, computed tomography (CT) is more widely available, is better suited for rapid screening in urgent settings, and can be used when patients have MRI exclusions (such as pacemakers). As such, many meningiomas are first encountered on CT scans obtained for different reasons. CT has a place in the diagnosis of meningioma because it is superior in demonstrating the effects of this neoplasm on adjacent bone, specifically osseous destruction in atypical or malignant meningiomas or hyperostosis associated with the benign meningiomas, and is more sensitive in detecting psammomatous calcifications in the tumor (seen grossly in approximately $25 \%$ of meningiomas). Benign meningiomas typically appear as rounded or elongated extraaxial masses that demonstrate a broad attachment to the dura. On CT, they are usually isodense, but can occasionally be hyper dense or slightly hypo dense compared to cerebrum.

Their extraaxial nature is suggested by a sharp interface with displaced brain parenchyma, the presence of a cerebrospinal fluid attenuation cleft and tumor intense enhancement. Meningiomas exhibit homogeneous attenuation prior and after administration of contrast material, but can show some heterogeneity depending on the consistency of tumor, i.e., the presence of calcium, fat, tumor necrosis. Hyperostosis of adjacent skull is highly suggestive of 
Fig. 1 Dural based mass (arrow in both figures) is appreciated in the left middle cranial fossa with associated hyperostosis of the sphenoid bone, squamosal temporal bone, orbital roof demonstrated by CT (a) and axial T2 MR sequence performed for surgical navigation (b). These findings are most consistent with an en plaque meningioma with involvement and associated hyperostosis of the underlying bone. Note the white matter vasogenic edema in the left temporal lobe due to mass effect (small arrow)
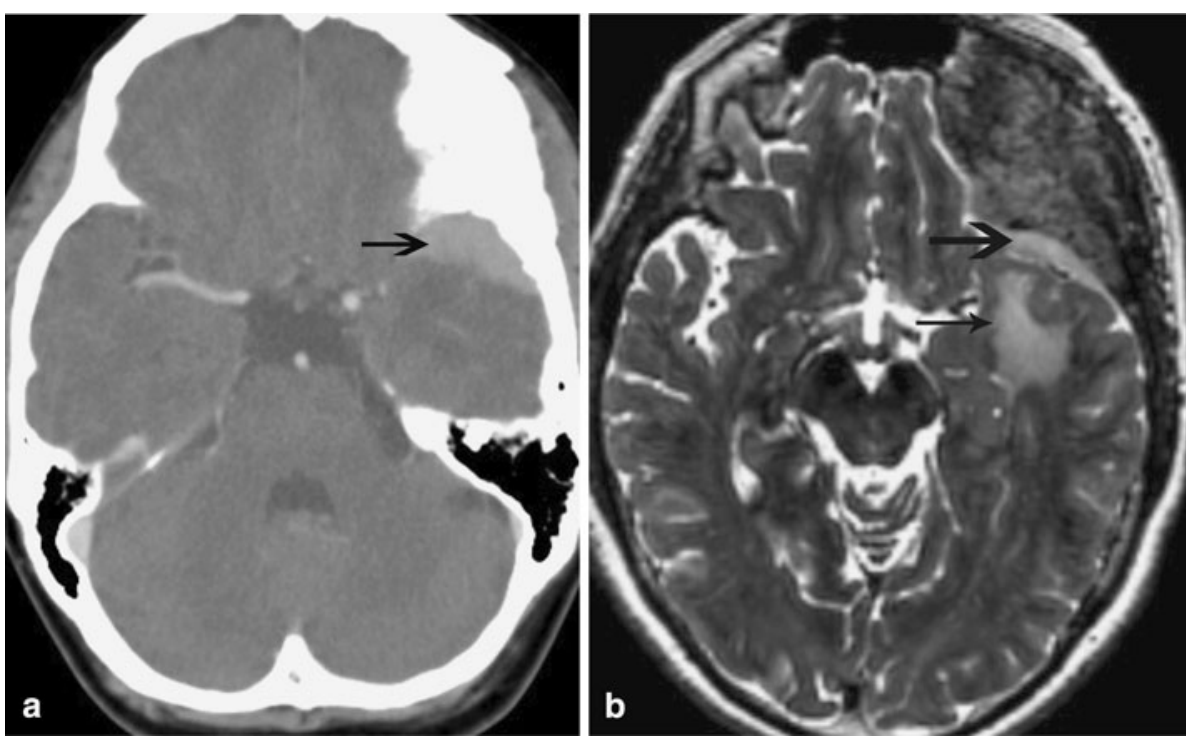

benign meningioma and is best demonstrated by CT, windowed on bone algorithm, as cortical thickening and hyper density (Fig. 1). Hyperostosis typically indicates infiltration of bone by meningioma [1].

\section{Magnetic resonance imaging}

Common imaging features of meningiomas on MRI

Most meningiomas have features that are similar including an extraaxial mass with signal intensity similar to cortex on T1 and T2 MRI sequences, avid homogeneous enhancement following administration of gadolinium contrast, and an enhancing "dural tail" which reflects neoplastic dural infiltration or reactive vascularity, or both, draining into the adjacent dura. Low signal intensity within the tumor may often be due to calcification or to vascular flow voids, a distinction sometimes difficult to make. Meningiomas can be nearly spherical or elongated (en plaque), multiple, and often take origin from a dural sinus, a feature important for surgical planning. These tumors also tend not to respect the dural boundary, which is a distinctive feature not typical of other neoplasms (Fig. 2).

Although most benign meningiomas are innocuous from the standpoint of metastatic potential, they may result in serious complications secondary to dural sinus invasion (Fig. 3), (with or without thrombosis), narrowing and thrombosis of significant arterial structures, and compression of cranial nerves and other important neural structures.

Edema associated with meningioma is thought to be vasogenic in origin, and probably related to tumor secretion of vascular endothelial growth factor (VGEF), rather than a result of direct mass effect on adjacent brain or venous

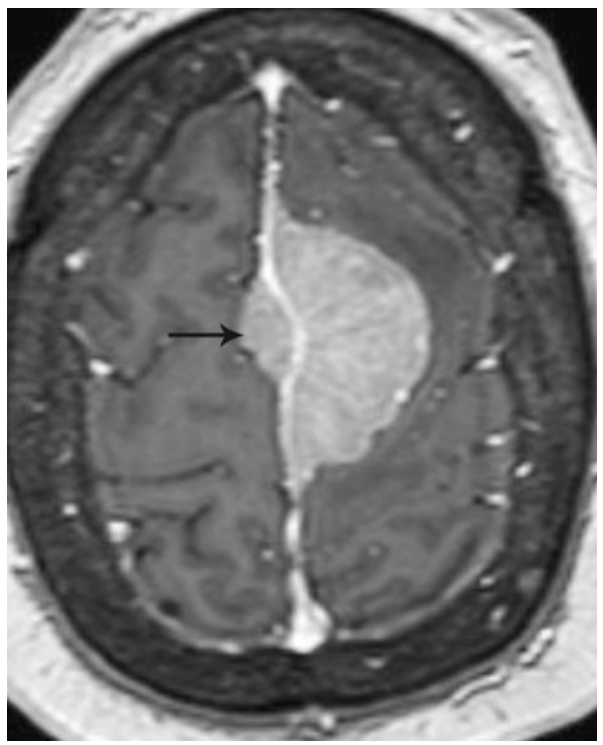

Fig. 2 Large interhemispheric extra-axial mass consistent with a parafalcine meningioma with a dominant left parafalcine component and a smaller right parafalcine component (arrow)

invasion causing vascular congestion [2]. The presence of intra-axial edema is said to predict an increased potential for recurrence $[3,4]$.

\section{Advanced imaging}

Nuclear medicine methods

There have been reports that radiolabeled agents, such as ${ }^{111}$ In-octreotide, that have an affinity for somostatin receptors can be useful in detecting and localizing meningiomas 

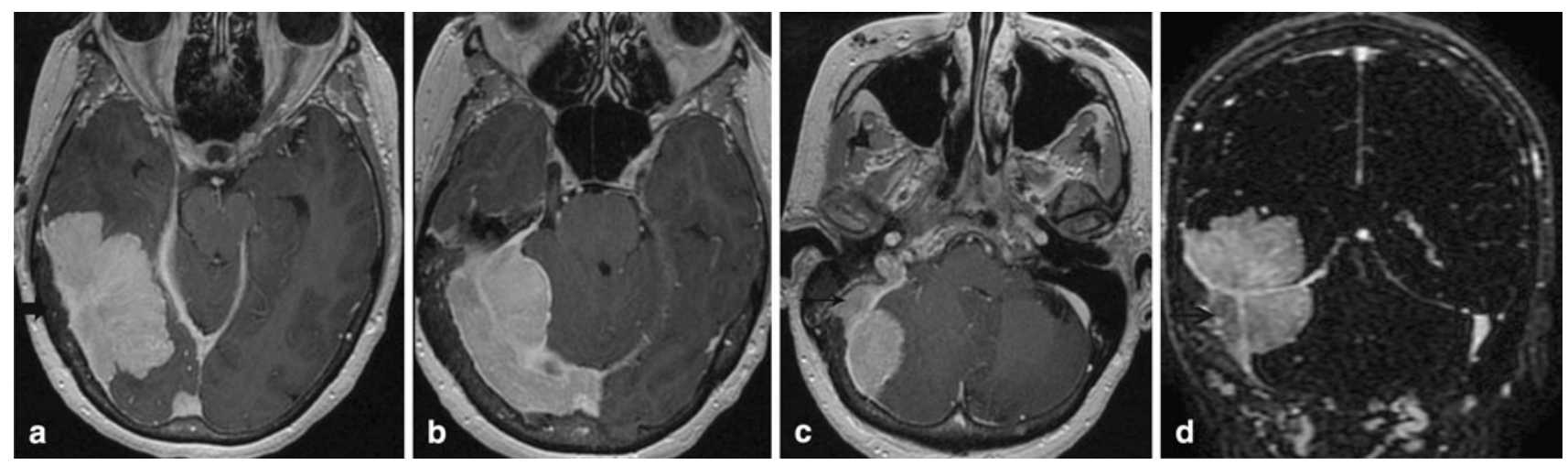

Fig. 3 Large right-sided transtentorial meningioma with growth into the right sigmoid (small arrow in c) demonstrated on post gadolinium axial $\mathrm{T} 1$ (a) and post gadolinium coronal MR venogram (d). Hyperostosis of adjacent skull pointed by large arrow (a)

[5]. However, this ability is countered by the relative lack of specificity in differentiating meningiomas from other lesions such as high-grade glioma or pituitary adenomas, among others. PET imaging is attractive for investigating the metabolic activity of tumors [6]. However, the role of PET imaging in the evaluation of meningiomas is complicated by the variable metabolic presentation in different meningioma types. In typical benign meningiomas, the usual metabolic marker F-18 fluorodeoxyglucose, presents with isometabolism on PET imaging. Malignant meningiomas may display hypermetabolism which confounds their differentiation from other intracranial tumors [7]. In general, nuclear medicine techniques provide capabilities that are sensitive for meningiomas but that have relatively low specificity.

\section{Diffusion MRI}

It is possible using MRI to sensitize the image appearance to the extent to which water can freely diffuse in any volume element (voxel). When the motion of water molecules within a voxel is restricted there is greater magnetization coherence and that voxel will appear bright. This technique is referred to as diffusion weighted imaging (DWI). Reduced water diffusivity (Fig. 4a) has been correlated with more aggressive tumor behavior and is sometimes seen with atypical/ malignant meningiomas, high cellular density, and recurrence [8].

The diffusion weighting in DWI acquisitions is encoded on top of the usual T1 and T2 properties of the underlying sequence. It is possible to create images that are insensitive to those underlying $\mathrm{T} 1$ and $\mathrm{T} 2$ values by performing multiple DWI acquisitions and extracting from them a map of the diffusion effect alone, referred to as an apparent diffusion coefficient (ADC) map. A decrease in ADC values (Fig. 4b) at follow up of a benign meningioma should raise suspicion for dedifferentiation to higher tumor grade [9]. Although diffusion-weighted imaging provides an added tool in the approach to defining meningioma grade a recent report [10] calls into question the predictive ability of DWI methods in grading meningiomas or identifying histological sub-types.
Fig. 4 Reduced diffusion is seen within this left parafalcine meningioma. First image (a) demonstrate high signal on the DWI, with corresponding low signal on the ADC maps (b)
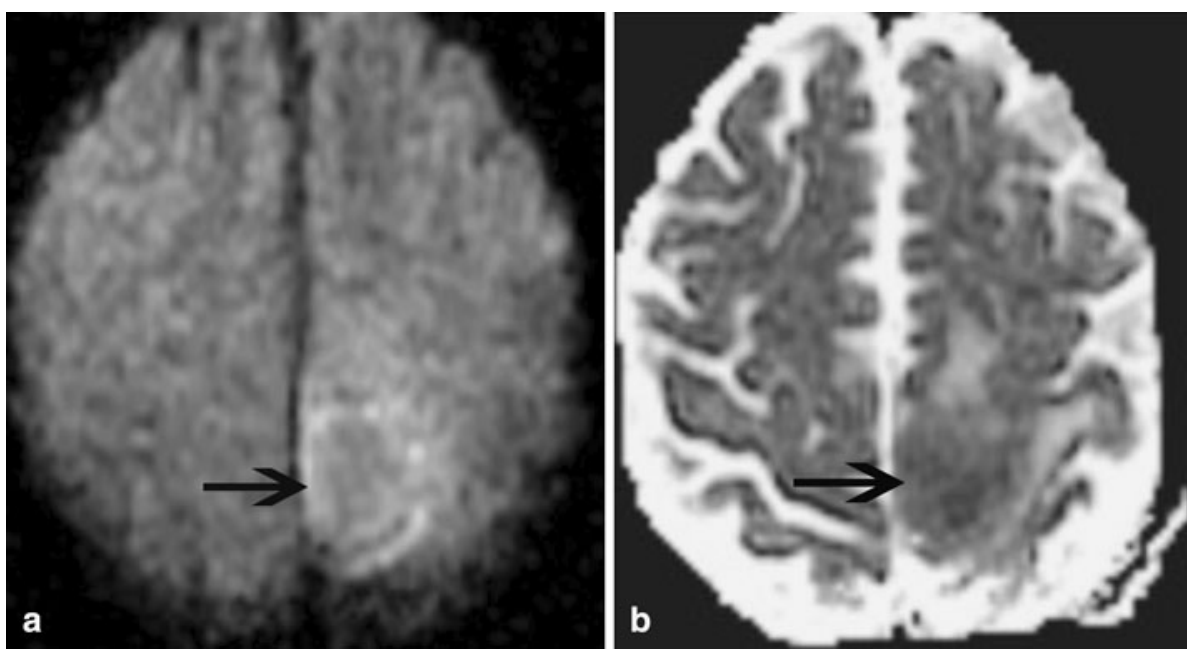


\section{Perfusion}

It is possible to perform MRI with acquisition times that are short enough to capture the changes in signal intensity as a bolus of contrast material passes through the brain. These methods, termed perfusion MRI, can provide useful information on the vascular supply of meningiomas, which is only implied from conventional MRI. Typically, the injection is performed at a rate and concentration that causes a loss of signal secondary to perturbation of the magnetic field by the contrast agent. The method used is an echo-planar contrast-enhanced $\mathrm{T} 2 *$ weighted sequence rapidly performed prior, during, and after the bolus infusion of gadolinium contrast material using an intravenous injection. The curves reflect the permeability between intravascular and extravascular compartments, as well as cerebral blood volume. The relative cerebral blood volume (rCBV) is measured within a tumor comparing to the contralateral normal white matter and can be displayed on color maps [11]. Perfusion curves provide additional prognostic information by helping distinguish between benign and atypical/malignant meningiomas.

Benign meningiomas typically derive their blood supply from the external carotid via dural branches. These vessels do not contain a blood brain barrier and are thus quite permeable to gadolinium, which is reflected by a curve with little or no return to baseline following infusion (Fig. 5a, b).

As the meningioma enlarges, it may parasitize pial branches from the brain parenchyma, which do contain a blood brain barrier. The perfusion scans will show an elevated cerebral blood volume with intensities that return to baseline signal levels, reflecting an intact blood-brain barrier of the internal carotid artery supply, as seen in Fig. 6. A high volume of pial-cortical supply (as opposed to dural-meningeal supply) usually predicts an aggressive meningioma with a higher tendency of recurrence.

The cerebral volume of peri tumoral edema was found by Zhang et al. [11] to be elevated surrounding malignant meningiomas compared to the vasogenic edema associated with benign meningiomas, and likely related to angiogenesis/microvascular proliferation in the peri tumoral brain.

\section{Conventional angiography and endovascular embolization}

Conventional angiography is most often performed for preoperative endovascular embolization and is intended to minimize the blood loss intraoperatively. With the increase use of preoperative embolization, the subsequent MRI changes and treatment complications [12], i.e., hemorrhage and necrosis sometimes present a confusing imaging picture for a radiologist who is unaware of the prior embolization procedure. MRI changes that occur after embolization of meningiomas usually include a decrease in gadolinium contrast enhancement (Fig. 7b), reduced diffusion of the devascularized segment of the tumor (Fig. 7c, d).

\section{Advanced MRI during endovascular embolization of meningiomas}

MR perfusion using intraarterial injections

As discussed above, MRI offers advantages over X-ray angiography in the evaluation of tissue physiology, including measurement of diffusion and perfusion characteristics that serve as proxies for tissue infarction and vascularity, respectively. In assessing the vascularity of meningiomas,
Fig. 5 MR perfusion of left frontal meningioma demonstrates significantly elevated relative cerebral blood volume (area under the curve is large) (purple curve in b), compared with contralateral normal matter (green curve). The less than $50 \%$ return to baseline (b) is typical of the lack of blood brain barrier of the external carotid artery
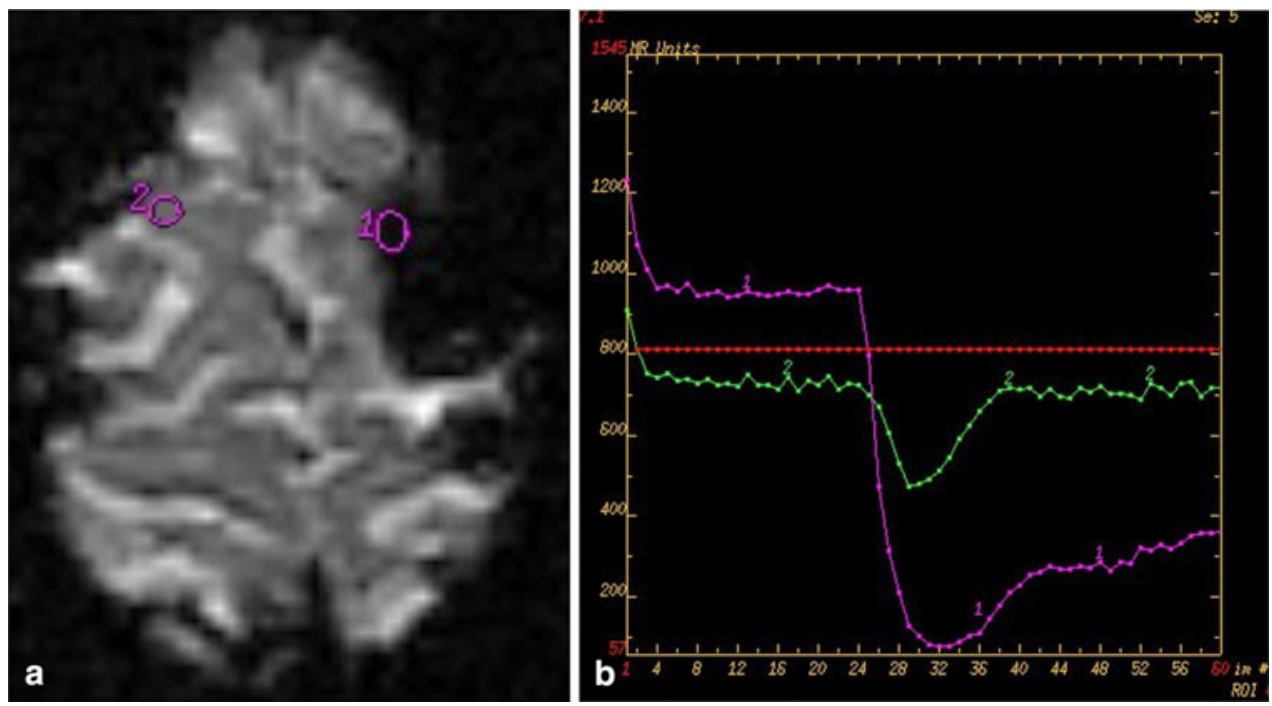
Fig. 6 Large ethmoid groove meningioma (a) with perfusion characteristics suggesting internal carotid artery supplyintact blood-brain barrier/no permeable vessels. ROI placed in the menignioma ( 1 in $\mathbf{c}$ ) is compared to normal white matter. A large area under the curve (d) represents elevated cerebral blood volume
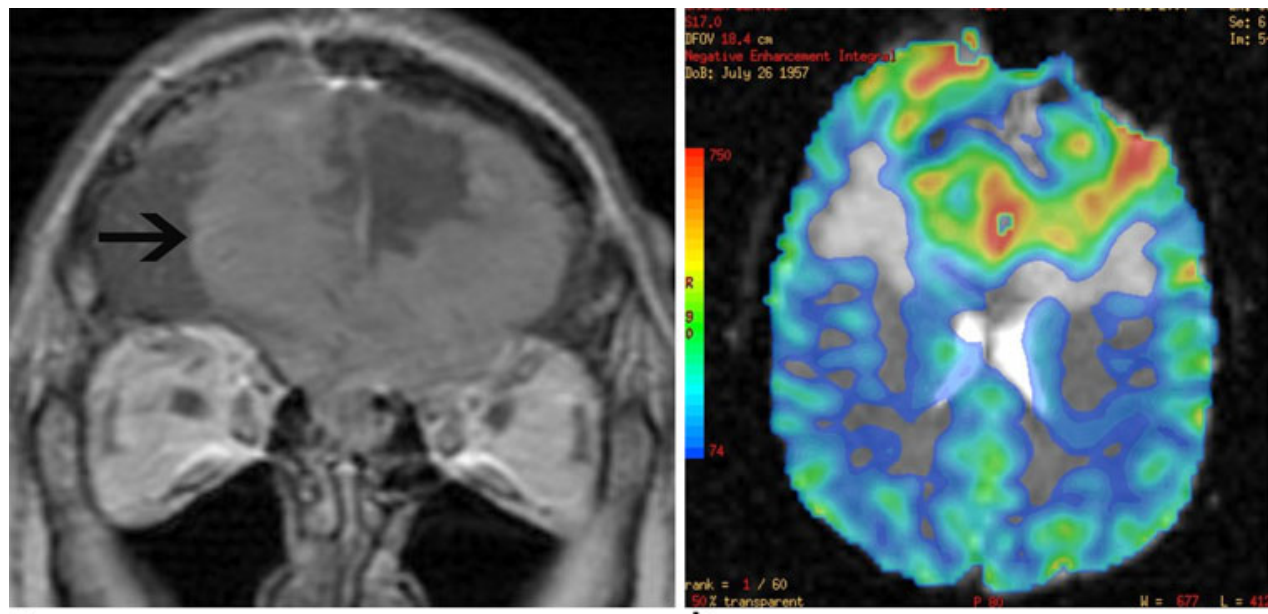

a

b
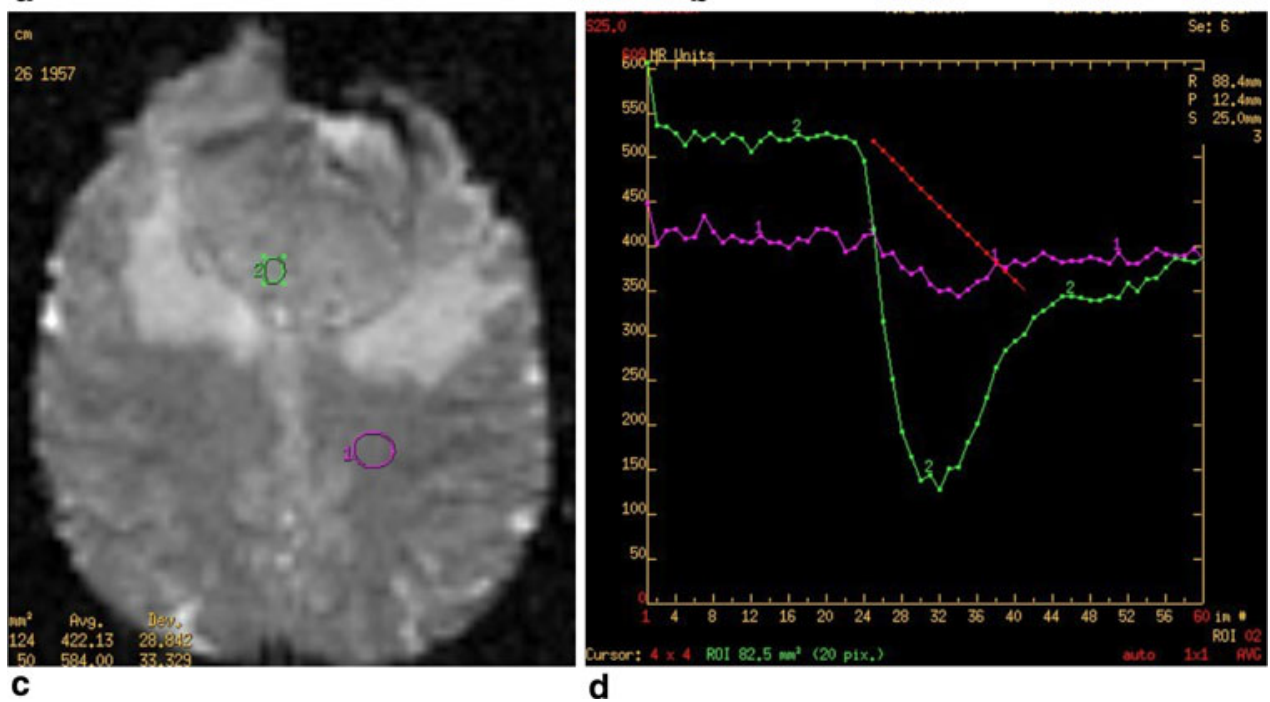

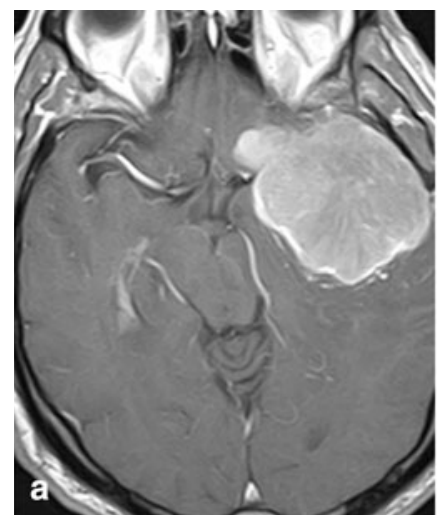

Fig. 7 Pre- (a) and post-embolization (b) T1 post gadolinium on same patient demonstrates an initially homogeneously enhancing meningioma with decreased enhancement, reduced diffusion (c) and low signal on the ADC map (d) in the embolized component. The

there is substantial interest in clearly defining which specific arterial branch is providing blood supply to the specific regions of interest. For example, preoperative embolization

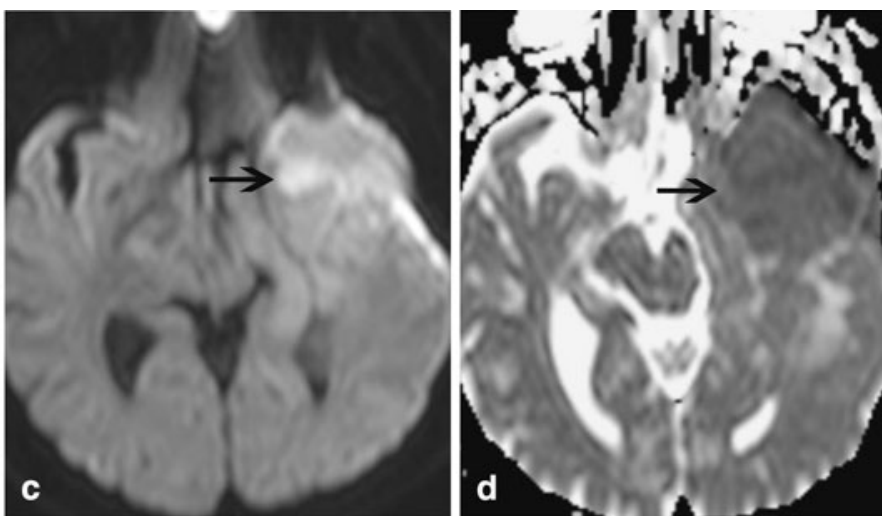

features accompanying embolization can mislead the radiologist or surgeon, if they are not provided the history of the embolization procedure

of intracranial meningiomas is performed to reduce tumor vascularity and thus minimize operative blood loss but is not possible if the internal carotid artery (ICA) is the only source 
of blood supply as embolic material delivered through the ICA would also obliterate normal brain tissue.

Conventional MR perfusion methods are performed with an intravenous injection into an arm vein. The contrast material is then transported back to the heart and ejected into the arterial system. As such, intravenous MR perfusion studies are non-selective and perfusion of a specific region of interest reflects supply from all arterial sources. Furthermore, the temporal passage of the contrast bolus is substantially modulated by significant patient-specific characteristic dynamics that have little to do with tissue perfusion, such as recirculation times in the veins and the lungs, mixing efficiency in the heart, and arterial tortuosity. Selective intraarterial injections performed in an MR suite provide powerful new capabilities in evaluating meningioma perfusion. The ability to investigate the impact of intraarterial procedures on the end organ is now available at a number of sites that have so-called XMR suites, an installation that contains both an X-ray fluoroscopy suite and an MRI scanner in the same room. Although it would not currently be recommended to perform catheterization solely for the purpose of determining perfusion characteristics, intraarterial MR perfusion studies are possible when conducted in the same session that the patient is already undergoing catheterization for pre-surgical embolization. Complete X-ray catheter cerebral angiography is performed in the context of preoperative embolization and, thus, fulfills a secondary goal of identifying all arterial supply to the tumor, whether embolized or not. X-ray angiography is able to qualitatively evaluate regional capillary-level vascularity within the tumor as each supplying artery is separately injected with iodinated contrast material.

At the University of California San Francisco, the XMR suite consists of a combined X-ray angiography and $1.5 \mathrm{~T}$
MRI suite in which a patient can be slid on a single bed between X-ray angiography and MRI intraprocedurally. That suite has been used to monitor the completeness of tumor embolization in 15 patients [13]. Via a standard transfemoral arterial approach under X-ray guidance nonbraided 5 French (Fr) diameter diagnostic catheters (which have undergone extensive testing for MR safety [14]) are placed in the external carotid artery (ECA) of subjects. Patients are then slid from X-ray to MR nd a baseline MR perfusion study is performed by injecting dilute gadolinium contrast through the ECA catheter. The catheter is then pulled back to the CCA and a similar intraarterial (IA) perfusion study is performed. By subtracting the ECA supply from the CCA supply, the ICA supply to the tumor can be determined without having to do a separate ICA catheterization. Patients are then moved back to the X-ray fluoroscopy suite for the embolization portion of the procedure. The intraarterial study can then be repeated postembolization (Fig. 8).

The technique of endovascular meningioma embolization is well established [15-18]. In brief, a microcatheter (1.9-2.3 Fr) is placed through the 5 Fr catheter in the ECA superselectively into the dural vessel supplying the tumor (usually the middle meningeal artery, a branch of the ECA) (Fig. 9). Microcatheter angiograms are performed to confirm that only tumor (and not normal critical structures such as the retina) is supplied by the catheterized vessel. Embolization is achieved by injection of $350-500 \mu \mathrm{m}$ diameter plastic particles (polyvinyl alcohol, PVA) until stasis is achieved in the tumor-supplying artery. The microcatheter is then cleared by saline injection and pushable platinum coils are often placed more proximally in the feeding artery to achieve complete arterial occlusion. The microcatheter is removed, but the $5 \mathrm{Fr}$ catheter remains in the ECA. The patient is then moved back into
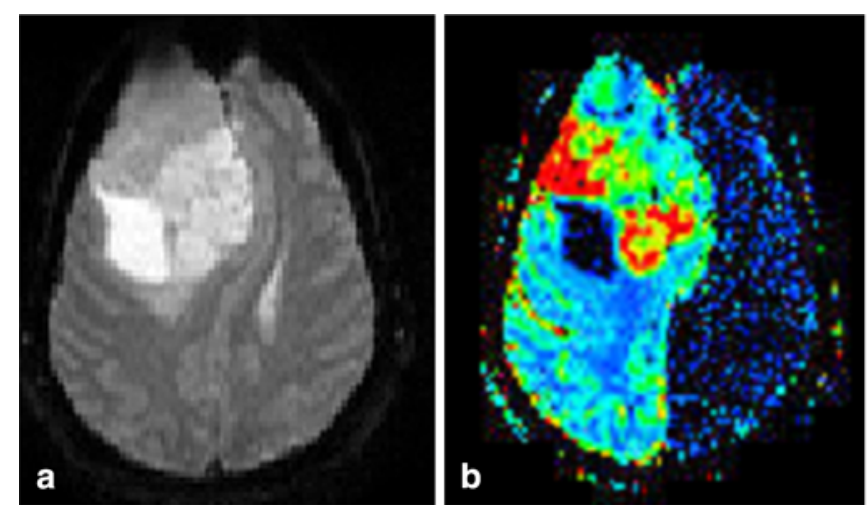

Fig. 8 (a) Meningioma visualized on MRI prior to treatment. (b) Intraarterial injection into the common carotid artery provides a map of Cerebral Blood Volume related to supply from ICA and ECA pre-embolization [note that normal brain tissue on the ipsilateral side also reflects perfusion effects]. (c) Pre-embolization intraarterial
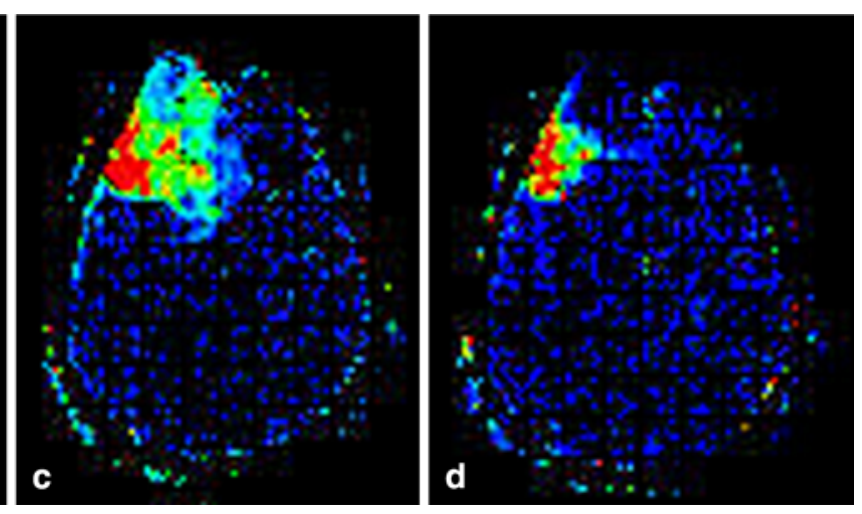

injection into the external carotid artery provides a map of Cerebral Blood Volume related to supply from the ECA alone [note no perfusion of normal brain tissue is noted]. (d) Post-embolization intraarterial injection into the ECA displays a small residual supply from the ECA that was not obliterated at embolization 


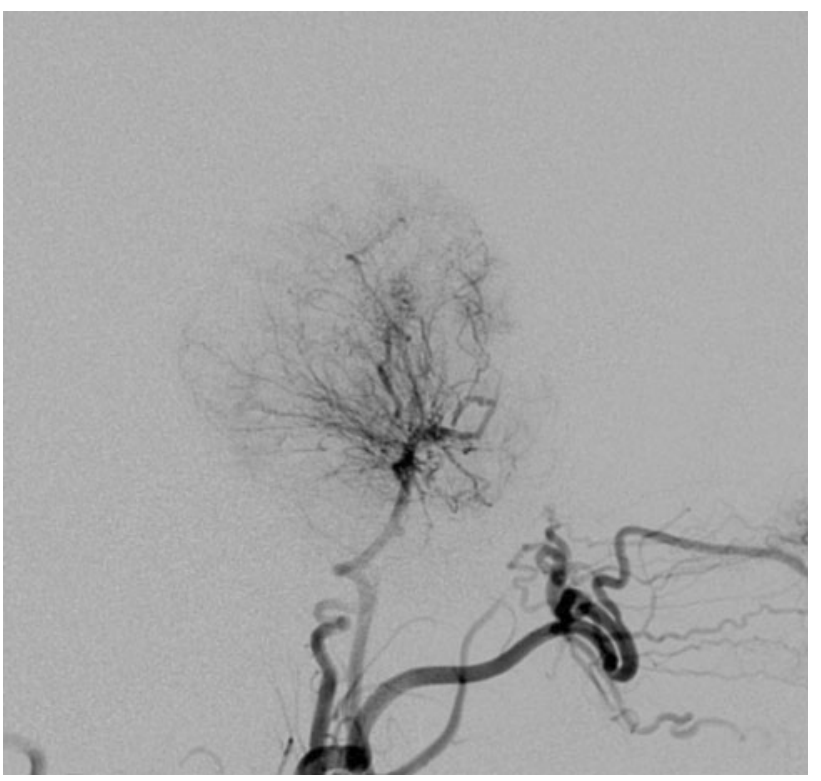

Fig. 9 Characteristic appearance at angiogram of a middle cranial fossa meningioma with extensive arterial supply from a left middle meningeal artery

the MR scanner and selective IA perfusion studies are repeated with the $5 \mathrm{Fr}$ catheter in the ECA and then pulled back into the CCA. After this second set of IA perfusion studies, the $5 \mathrm{Fr}$ catheter is removed and the femoral arterial access site is closed.

The advanced MRI techniques that are used intraprocedurally during the course of endovascular embolization of meningiomas show promise for guiding both embolization and surgery [13]. Different IA perfusion techniques, including $\mathrm{T} 2 *$ dynamic susceptibility contrast (DSC) and T1 dynamic contrast enhanced (DCE), have been applied. As expected, T2* based techniques appear to be of limited value in heavily calcified meningiomas, but $\mathrm{T} 1$ based techniques may be of more use in such lesions. MR perfusion maps appear to be more sensitive in detecting residual tumor vascularity as compared to qualitative analysis of X-ray angiographic images [13]. ECA perfusion usually correlates well with dural tumor supply on X-ray angiography; ICA perfusion (CCA minus ECA) similarly usually correlates with pial supply. Diffusion-weighted imaging (DWI) is also being applied to evaluate for embolization-induced tumor infarction (or unanticipated ischemia of non-tumor tissue). Correlations between MR findings and regional histology of resected tumors are performed to validate MR findings but study numbers are not yet sufficient to define which combination of advanced MR techniques is optimal.

It is hoped that IA perfusion techniques will provide better preoperative insight into tumor vascularity, allowing surgeons to better anticipate which areas of tumor are still likely to bleed intraoperatively when embolization is incomplete. These techniques may also prove valuable in monitoring the effects of new embolic agents, such as liposomes carrying chemotherapeutics or anti-tumor antibodies. As minimally invasive MRI of tumor physiology improves and is validated against resected tumor histology, its ability to monitor nonsurgical treatments for dural and nondural brain neoplasms also comes closer to realization.

\section{Conclusion}

In summary, meningiomas have a typical but sometime variable appearance on MR and CT. Modern imaging tools can usually suggest the histological diagnosis, but usually not the grade of tumor. Perfusion and diffusion imaging have been useful tools for diagnosis and for suggestion of alternative histologies, as well as predicting aggressive histological features. Catheter angiography performed during preoperative embolization of meningioma is useful in elucidating the feeding arteries of the meningioma. There are now investigative studies that indicate that intraarterial MR perfusion methods could be useful in better understanding the perfusion characteristics of meningiomas, and could be used in montoring the delivery of therapeutics.

Acknowledgment This work has been supported by grant CA123840 from the National Institutes of Health.

Open Access This article is distributed under the terms of the Creative Commons Attribution Noncommercial License which permits any noncommercial use, distribution, and reproduction in any medium, provided the original author(s) and source are credited.

\section{References}

1. Pieper DR, Al-Mefty O, Hanada Y, Buechner D (1999) Hyperostosis associated with meningioma of the cranial base: secondary changes or tumor invasion. Neurosurgery 44(4):742-746, (discussion 746-747)

2. Pistolesi S, Fontanini G, Camacci T et al (2002) Meningiomaassociated brain oedema: the role of angiogenic factors and pial blood supply. J Neurooncol 60(2):159-164

3. Vignes JR, Sesay M, Rezajooi K, Gimbert E, Liguoro D (2008) Peritumoral edema and prognosis in intracranial meningioma surgery. J Clin Neurosci 15(7):764-768

4. Simis A, Pires de Aguiar PH, Leite CC, Santana PA Jr, Rosemberg S, Teixeira MJ (2008) Peritumoral brain edema in benign meningiomas: correlation with clinical, radiologic, and surgical factors and possible role on recurrence. Surg Neurol 70(5):471-477

5. Krenning EP, Breeman WA, Kooij PPM et al (1989) Localisation of endocrine-related tumours with radioiodinated analogs of somatostatin. Lancet 4:242-244

6. Chang AS, Ross JS (2008) Diagnostic neuroradiology: CT, MRI, fMRI, MRS, PET, and Octreotide SPECT. In: Lee JH (ed) Meningiomas, diagnosis, treatment and outcome. Springer, Heidelberg, 55-65 
7. Lippitz B, Cremerius U, Mayfrank L et al (1996) PET-study of intra-cranial meningiomas: correlation with histopathology, cellularity and proliferation rate. Acta Neuroschir Suppl 65:108-111

8. Nagar VA, Ye JR, Ng WH et al (2008) Diffusion-weighted MRI: diagnosing atypical or malignant meningiomas and detecting tumor dedifferentiation. AJNR Am J Neuroradiol 29(6): $1147-1152$

9. Hakyemez B, Yildirim N, Gokalp G, Erdogan C, Parlak M (2006) The contribution of diffusion-weighted MRI to distinguishing typical from atypical meningiomas. Neuroradiology 48(8): 513-520

10. Santelli L, Ramondo G, Della Puppa A, Ermani M, Scienza R, d'Avella D, Manara R (2010) Diffusion-weighted imaging does not predict histological grading in meningiomas. Acta Neurochir (Wien) 152(8):1315-1319

11. Zhang H, Rödiger L, Shen T (2008) Perfusion MRI for differentiation of benign, malignant meningiomas. Neuroradiology 50(6):525-530

12. Carli DF, Sluzewski M, Beute GN, van Rooij WJ (2010) Complications of particle embolization of meningiomas: frequency, Risk Factors, and Outcome. AJNR Am J Neuroradiol 31:152-154
13. Martin AJ, Cha S, Higashida RT, Cullen SP, Halbach V, Dowd CF, McDermott MW, Saloner DA (2007) Assessment of meningioma vasculature and the effects of embolization with intra-arterial MR perfusion imaging. Am J Neuro Radiol 28: 196-205

14. Martin AJ, Baek B, Acevedo-Bolton G, Higashida R, Comstock J, Saloner D (2009) MRI during endovascular procedures: an evaluation of the potential for catheter heating. Magn Reson Med 61(1):45-53

15. Hieshima GB, Everhart FR, Mehringer CM et al (1980) Preoperative embolization of meningiomas. Surg Neurol 14:119-127

16. Latchaw RE (1993) Preoperative intracranial meningioma embolization: technical considerations affecting the risk-to-benefit ratio. AJNR Am J Neuroradiol 14:583-586

17. Dean BL, Flom RA, Wallace RC et al (1994) Efficacy of endovascular treatment of meningiomas: evaluation with matched samples. AJNR Am J Neuroradiol 15:1675-1680

18. Chun JY, McDermott MW, Lamborn KR et al (2002) Delayed surgical resection reduces intraoperative blood loss for embolized meningiomas. Neurosurgery 50:1231-1235 discussion 1235-1237 